Marta Fernández-Villanueva and Konstanze Jungbluth (eds.), 2o16. Beyond Language Boundaries. Multimodal use in Multilingual Contexts. [264] pages. Berlin, Boston: De Gruyter. ISBN 978-3-11-045640-0 €99.95 / US\$140.00 / G BP 91.00.

\title{
Summary
}

The peer-reviewed volume 'Beyond Language Boundaries. Multimodal use in Multilingual Contexts', edited by Fernández-Villanueva and Konstanze Jungbluth aims to approach theoretical and empirical topics surrounding multimodality and multilingualism from a novel and innovative point of view. The anthology's structure consists of an introduction, and two themed parts presenting a total of thirteen papers. In the short introduction, the editors outline the central concepts of the issue. After presenting their projects and affiliations, which were central in bringing the authors of the chapters together, they make the reader aware of the areas that are discussed and give an overview of the languages that are dealt with in the different chapters, which investigate language use in Europe and America. Thereby three different perspectives on the topic of the book are mentioned - 'research on multimodality, on second language (L2)/foreign language acquisition and on plurilingual language use performed by multilingual speakers' (Fernández-Villanueva, Jungbluth 2016: p.V). Providing a contextual and methodological overview (gestures, language contact and multimodal aspects into grammar; pragmatic aspects; language use and acquisition including code-switching; discourse markers and argument structure; deixis, co-construction; acts of identity) they group the authors of the articles accordingly and present a volume that will interest a broad audience of linguists from different disciplines. As a connecting link, the editors mention that not only do all of the chapters deal with multimodal language use in multilingual contexts, but that all of them also draw upon empirically collected corpus data. Subsequently, they discuss the organization of the book and briefly summarize the various authors' papers, before concluding with the central questions they aim to answer.

After the introduction, the book is structured in two parts, the first part being 'Multimodal Language Use' with six topical papers, the second part 'Language Use in Multilingual Contexts' presenting seven papers and concludes with an index of frequently used terminology and the endnotes.

In the first part, the discussion is opened with a chapter from Alturo, Ignasi and Payrató, elaborating the usefulness of 'Functional Discourse Grammar' to approach multilingual and multimodal competences of speakers. Within their structuralist approach, they strongly argue for the integration of gesture and

(C) MIRIAM WEIDL, 2019 | DOI:10.1163/19552629-01202010

This is an open access article distributed under the terms of the prevailing CC-BY-NC License at the time of publication. 
verbal signs into grammar analysis, which they support with various examples, mainly from Spanish and Catalan but also providing data from many other languages.

Following a different, but still structuralist approach, Tessendorf concentrates on the various pragmatic aspects and functions of recurrent hand gestures in German and Spanish monolingual language use. She discusses the many different functions of gestures in everyday conversations but also in relation to the structure of speech, which she concludes can be grouped around topics, according to the culture or speech community.

In the third chapter, Yepes aims to develop a methodological strategy to particularly focus on the relationship of propositional and interactional variations with a focus on the dynamic verbs 'gehen' [go] and 'kommen' [come] in monolingual German conversations of adult speakers from elicitation tasks. Using a multimodal corpus, which allowed the analysis of verbal and gestural deixis, he describes on the one hand a propositional level on the basis of morphosyntactic and semantic units, and on the other hand deictic alternation on an interactional level.

Dealing with a similar framework considering the central languages, Schmidt provides a discussion on proxemics in the field of physical contact situations in two separated cultural frames. She analyses non-verbal behavior in a comparative study of two television series originating from Spain and Germany, which are seen as partially representative for Spanish and German culture. Thereby she especially focuses on physical contact situations during greetings and farewells, using these to then discuss the implications for intercultural communication as well as the importance of an awareness of nonverbal communication for language learning to avoid misconceptions of nonverbal behavior patterns and communication breakdown.

The study carried out by Isaeva and Fernández-Villanueva examined the multimodal process of embodied communication, where emphatic and pointing hand gestures are used to perform discursive and interactional functions in case of lexical access difficulties during the speech event. Thereby they bring together some of the approaches already covered separately in the former papers. Analyzing German communications of Spanish/Catalan speakers who study German as a second language and represent differing levels of proficiency, they show that hand gestures actively participate as problem-solving components and therefore also decrease with improved proficiency of the speakers.

Thematically interrelated with the previous chapter discussing German teachers' identities in discursive interactions in Barcelona, i Puigdelliura and 
Fernández-Villanueva conclude this fist part of the volume. They focus on multilingual and multimodal cues within the semiotic dimensions like codeswitching, lexical inserts, gestures and facial expressions. Conducting multilingual quantitative semi-narrative interviews with teachers who either originate from Germany or Spain, their identifying positions were analyzed. Finally, they clearly show that the native and non-native speaker dichotomy still exists in their context and point out that non-verbal speech as well as multimodal features and multilingual practice are hugely insightful in identity research and the categorization of membership and dynamic positioning of individuals.

Opening the debate on language use in multilingual context in the second part of the volume, Jungbluth reviews existing literature using examples published by various scholars to draw on co-constructions in multilingual settings. Therefore, she discusses co-constructing in communication in a broader and narrower sense, looking at semantic, lexical and pragmatic co-constructions of speakers, including collaborate voiced speech acts. Starting with monolingual data, she then provides multilingual and multimodal examples in an attempt to bridge contexts and deliver insights into communication beyond language boundaries and calls attention to the lack of in-depth investigations in research so far.

Da Milano's paper builds up on two case studies from multilingual Catalans living in Spain and multilingual Italians who migrated to Spain to analyze their expressions of personal deixis, in relation to spatial and temporal deixis. As referring to oneself and also to another individual (person reference) is considered one of the first indicators of an individual's personality, Da Milano especially concentrates on pronouns which prove powerful tools that disclose information on cultural identity.

Next, Mestre and Cuenca present an interesting discussion on the use of connectives (conjunctions or parenthetical connectives) indicating binary relationships with argumentative functions, while also considering frequency as well as the cause-effect relationship. Therefore, they use a corpus from Spanish parliamentary debates, which, from a linguistic and ideological point of view, is bilingual in Catalan and Spanish. They present results demonstrating how differently binary connectives are used in Spanish and Catalan, but further indicate that (also) the individual style needs to be understood as a key factor for the use of binary connectives.

The subsequent chapter concentrates on the fluid identity construction of two multilingual and multicultural speakers with different backgrounds but shifts the perspective in the direction of language attitudes. Through videorecorded oral questionnaires and semi-structured interviews, Peters analyses 
the participants' self-reports with a focus on affective and epistemic attitudes, metalinguistic and metacommunicative awareness. First exemplifying language attrition, Peters then presents the construction of unique individual and social identities that are marked by the change of languages and linguistic environment as defining characteristics for identity.

Within a more theoretical approach, Rhobodes discusses the structural aspects of language mixing phenomena on the basis of German and Polish language mixing. Like transitions and passages, the non-static character of the languages enables the transfer of grammatical features between the languages, but also shows context-dependency. The author stresses the importance of an interdisciplinary approach (integrating phonetic, morphological and syntactic aspects) in the investigation of language contact induced language mixing and its structural consequences where an opening, crossing and dissolving of language borders is possible.

Through the presentation of three case studies, Haid investigates the construction of identity and analyses the creation of community through codeswitching into the language of the masses. Therefore, political speeches of well-known international politicians presenting in front of a foreign audience are the center of the investigation, who codeswitch in a targeted and prepared manner. Thereby, codeswitching is used as a political and rhetorical instrument that creates solidarity with the listeners and, since in all of the examples the strategy was successfully implemented, resulted in a positive response of the audience, creating emphasis and solidarity.

In the final chapter, Collins discusses the effect and hindrance of being a first language speaker of English, embodying the identity of a native speaker, upon second language learning on a local level. Considering transnational and expatriate features, he presents four exemplifying speakers who relocated from Ireland to Luxembourg in their adult age, showing that the high communicative value of English induces a reduced probability to learn a second language.

\section{2 Evaluation}

Fernández-Villanueva and Jungbluth deal with a considerable variety of matters, as the broad/inclusive title 'Beyond Language Boundaries. Multimodal use in Multilingual Contexts' implies. Introducing the contributions to this volume, the authors refer to a coherent line in the book that is represented through the overlapping concepts of multilingualism and multimodality in language use. Even though I definitely agree on their interlacement, remarks 
need to be made. Primarily, the introduction does present us with central topics, however, unfortunately neither the term multimodality nor multilingualism are further defined or discussed (see e.g. Blommaert and Backus, 2012) with reference to existing literature, which in my view presents a difficulty. However, some of the published papers sufficiently discuss terminologies central to their research.

All of the discussed data in the volume presents speech events, in which multimodality is an (almost) ever-present phenomenon and cannot be omitted (see e.g. Norris, 2012; Norris and Maier, 2017). Simultaneously, multiple languages can be applied, representing the multilingualism they mention in their title. From my perspective, all of the data presented could be seen as multimodal and multilingual, but only within a very open post-structuralist approach that does not recognize categorizations of languages (see e.g. Blommaert, Collins, and Slembrouck, 2005; Dewaele and Wei, 2012; Garcia and Wei, 2014 for some innovative approaches), which the title 'Beyond Language Boundaries' (that unfortunately remains uncommented on in the introduction) might permit. However, looking at the chapters, we are presented with a different reality, as only Rhobodes discusses language borders between two languages to a certain extent. Since most of the authors seem to have worked together over a longer period of time (as mentioned in the introduction) their shared views on extensive terms that allow various interpretations would have been particularly interesting.

Most of the papers follow the aim of jointly approaching multimodality and multilingualism in their fields (Alturo, Clemente and Payrató; Isaeva and Fernández-Villanueva; i Puigdelliura and Fernández-Villanueva; Jungbluth; Peters; Haid) and open a new and innovative debate around some important theoretical and empirical questions. Other scholars clearly discuss language as closed entity focusing on the distribution of different multimodal signs but, in my opinion, do not touch upon the topic of multilingualism (Tessendorf; Yepes; Schmidt). On the other hand, some papers in the second part of the book only focus on multilingualism (Da Milano, Mestre and Cuenca, Peters and Collins) without considering or mentioning multimodality. Structurally, the papers that focus on multimodality, multilingualism or both of them were presented mixed, which created some sense of disorientation while reading the volume. However, if it was meant to demonstrate that multimodality is seen as an indicator that automatically makes individuals (or the society?) multilingual (similar to the view that different lects, varieties and styles within one defined language can be seen as multilingualism) and vice versa, this could have been clearly stated. 
The editors endeavor to contribute to a complex and manifold field, with a Western, Euro-centric view on well-described languages but still demonstrate the diversity of multimodality and multilingualism present, which make the book in that sense coherent and cohesive. However, an impression remains that the title of the book as well as the structuring of the papers might have been adapted and discussed in a different way to facilitate a theoretical and disciplinary placement for the readers, for example focusing on the topics they refer to in the introduction as a starting point.

Theoretical and methodological approaches for analysis of multimodality and multilingualism can, as this volume clearly shows, strongly diverge from each other. This opens many questions that are worth further investigation, including a field to (re-)discuss existing terminologies from a different point of view. For myself, the volume especially aroused my interest in longitudinal studies presenting the development of multimodal signs in multilingual language use and further raised questions concerning how multimodal signs are applied in multilingual language use in settings that are not that well described. Ultimately, this edited volume will appeal to and interest a wide variety of readership and is most appropriate for professionals and researchers.

\author{
Miriam Weidl \\ soAs, University of London \\ mw65@soas.ac.uk
}

\title{
References
}

Blommaert, Jan, and Ad Backus. 2012. Superdiverse Repertoires and the Individual. In I. de Saint-Jacques and J.-J. Weber (Eds.), Multimodality and Multilingualism: Current Challenges for Educational Studies (pp. 1-33). Rotterdam: Sense Publisher.

Blommaert, Jan, James Collins, and Stef Slembrouck. 2005. Spaces of Multilingualism. Language and Communication 25(3) (sPEC. Iss.): 197-216.

Dewaele, Jean-Marc, and Li Wei. 2012. "Multilingualism, Empathy and Multicompetence." International Journal of Multilingualism 9(4):352-66.

Garcia, Ofelia, and Li Wei. 2014. Translanguaging. Language, Bilingualism and Education. Basingstoke: Palgrave Macmillan.

Norris, Sigrid. 2012. Multimodality in Practice: Investigating Theory in Practicethrough Methodology Multimodality in Practice: Investigating Theory in Practicethrough Methodology. London: Routledge.

Norris, Sigrid, and Carmen Daniela Maier. 2017. "Multimodal Theory and Methodology: What Are Their Facets ?" In Interactions, Images and Texts: A Reader in Multimodality. Berlin: De Gruyter Mouton. 